\title{
Phonon hydrodynamics in frequency-domain thermoreflectance experiments
}

\author{
A. Beardo $\odot,{ }^{1, *}$ M. G. Hennessy $\odot,{ }^{2}$ L. Sendra $\odot,{ }^{1}$ J. Camacho, ${ }^{1}$ T. G. Myers,${ }^{3,4}$ J. Bafaluy $\odot,{ }^{1}$ and F. X. Alvarez ${ }^{1}$ \\ ${ }^{1}$ Physics Department, Universitat Autònoma de Barcelona, 08193 Bellaterra, Catalonia, Spain \\ ${ }^{2}$ Mathematical Institute, University of Oxford, Andrew Wiles Building, Radcliffe Observatory Quarter, \\ Woodstock Road, Oxford OX2 6GG, United Kingdom \\ ${ }^{3}$ Centre de Recerca Matemàtica, Campus de Bellaterra, Edifici C, 08193 Bellaterra, Catalonia, Spain \\ ${ }^{4}$ Mathematics Department, Universitat Politècnica de Catalunya, 08028 Barcelona, Catalonia, Spain
}

(Received 8 October 2019; revised manuscript received 13 January 2020; accepted 27 January 2020; published 7 February 2020)

\begin{abstract}
The hydrodynamic heat transport equation with appropriate boundary conditions and $a b$ initio calculated coefficients is validated by comparing the corresponding analytical and numerical solutions with frequencydomain thermoreflectance experimental measurements in silicon. Special attention is devoted to identifying the resistive effects appearing at the interface between the metal transducer and the silicon substrate. We find that a Fourier model using frequency-dependent effective thermal conductivity cannot simultaneously explain the experimental phase shifts and the amplitude of the temperature oscillations, whereas the hydrodynamic model using intrinsic parameters provides good agreement across a wide temperature range. In addition, phenomenology appearing at reduced length and time scales in this kind of experiment at different temperatures is shown. Specifically, we find hydrodynamic modes of thermal transport that are analogous to pressure- and shear-wave propagation in viscoelastic media.
\end{abstract}

DOI: 10.1103/PhysRevB.101.075303

\section{INTRODUCTION}

It is well known that extracting thermal energy from semiconductor samples with reduced characteristic sizes is more difficult than from bulk materials [1-6]. This is indirect evidence that Fourier's law is not valid at these scales. In previous works, an effective form of this equation with a reduced thermal conductivity $[3,7-10]$ or a size-dependent thermal boundary resistance $[4,11,12]$ has been used to interpret experimental observations of size effects. A paradigmatic example in which this approach has been applied are time-domain and frequency-domain thermoreflectance (TDTR/FDTR) experiments in which an oscillating laser heats a metal thin film (the transducer) on top of a semiconductor substrate and the temperature evolution of the transducer is obtained by thermoreflectance techniques [2,13-18]. Specifically, these measurements have been interpreted as a way to measure the phonon mean free path spectrum and the accumulated thermal conductivity of the phonon modes in the substrate (because of the suppression of the modes with mean free paths larger than the thermal penetration depth).

However, detailed analysis of the temporal and spatial information provided by these experiments reveals the inadequacy of Fourier's law even in an effective form [2,12$15,19,20]$, and brings to light the need for refined transport equations that are based on parameters that are independent of the heating frequency and the geometry of the system. Obtaining and validating new equations able to describe these experiments opens the door to the development of new

*Corresponding author: albert.beardo@uab.cat technological applications aimed at improving the thermal management of electronic devices.

One of the recent proposals to describe the experimentally observed phenomena is phonon hydrodynamics [5,21,22]. This model has been used extensively to describe thermal properties in graphene and other two-dimensional (2D) materials where normal phonon collisions are dominant [23-25]. The conservation of momentum in such collisions influences the thermal response of the systems, which can be more accurately described with the use of the Guyer and Krumhansl equation (GKE) [26]. The same model equation has also been used to describe geometry effects in situations where normal collisions are not dominant [5]. The reason behind this is that momentum is also conserved at reduced length scales, this time due to the lack of collisions, and so the same macroscopic description of the thermal behavior can be employed.

The first direct evidence that Fourier's law cannot predict heat transport at the nanoscale was obtained in a recent work, where it was demonstrated that it could not describe the temperature profile near a nanoheater line, even using an effective thermal conductivity [5,21]. In the same work it was shown that changing to the GKE allows a better description of the measurements. Here we present further evidence using FDTR measurements. We show that an interpretation of this experiment in terms of Fourier's law leads to contradictory results that are resolved through the use of the kinetic collective model (KCM) $[21,22]$. The KCM is based on a macroscopic generalized heat transport equation with the form of the GKE with $a b$ initio calculated coefficients and appropriate boundary conditions. Specifically, we focus on the available experimental data from FDTR experiments [13,14]. The numerical solution of the transport equations using 


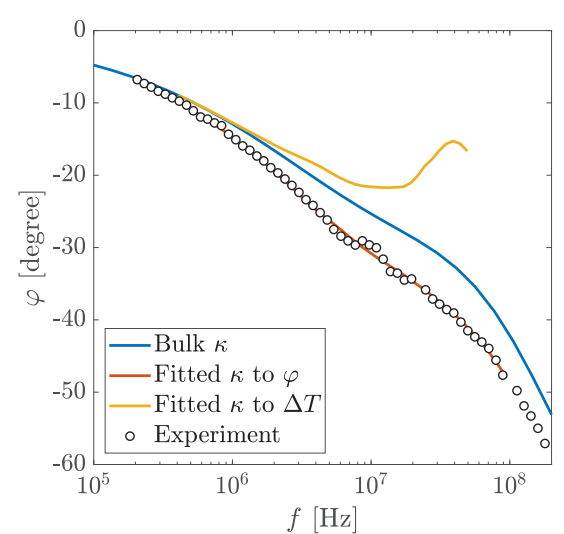

(a)

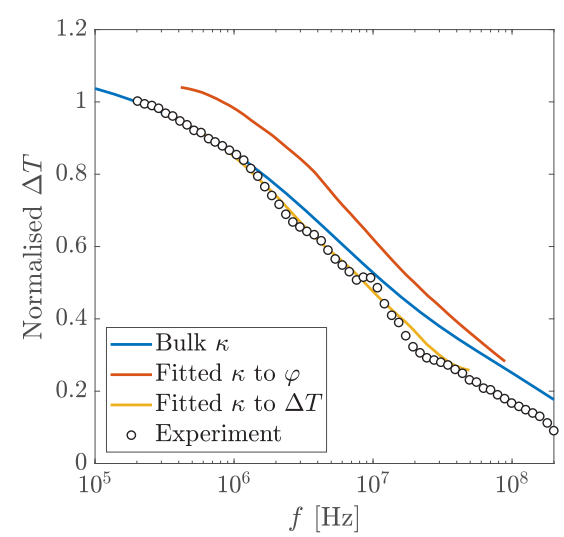

(b)

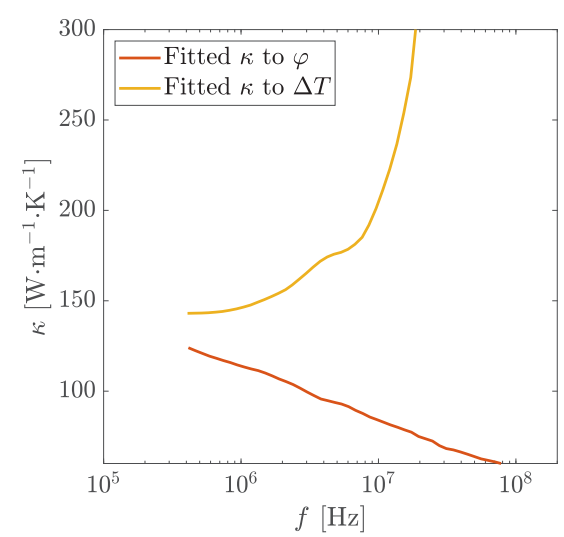

(c)

FIG. 1. (a) Phase shift $\varphi$ and (b) normalized temperature oscillation amplitude $\Delta T$ as functions of the heating frequency $f$ at $T_{0}=311 \mathrm{~K}$. Curves denote predictions from effective Fourier models based on bulk and fitted values of the substrate thermal conductivity, the latter of which are shown in panel (c).

finite-element methods (FEMs) allows a direct comparison with the experimental measurements. We show that the KCM properly describes the observed surface temperature phase shift with respect to the laser heating pulse and also the amplitude of the temperature oscillations by using $a b$ initio coefficients, i.e., all the equation parameters are independent of the geometry and heating frequency.

Furthermore, we derive analytical solutions to the KCM equations [22] for the case under study and compare with Fourier (bulk) predictions in order to gain phenomenological insight and interpretation of the process of heat release from metal domains to a silicon substrate. In particular, by using an analogy with the pressure and shear wave propagation in viscoelastic media, we distinguish $3 \mathrm{D}$ hydrodynamic heat transport mechanisms that cannot be observed in the Fourier framework.

Finally, we use the model to understand the effects that a transducer-semiconductor interface thermal boundary resistance (TBR) has on heat propagation. In contrast to previous interpretations in terms of the effective Fourier model $[4,13,14]$, which required ad hoc enhanced TBR, the present model uses a lower TBR that can be related with other theoretical models such as the diffuse mismatch model (DMM) [27] because of the inclusion of complementary intrinsic resistive effects arising from the non-Fourier heat dissipation in the substrate.

The article is organized as follows: In the second section, we motivate the use of generalized heat transport equations in this type of experiment by considering the nature of the predictions obtained from an effective Fourier framework. In the third section, we provide a description of the KCM including all the required boundary conditions. In the fourth section, we compare the experimental data with the numerical and analytical results from the KCM. In the fifth section, we discuss the applicability of the model to this type of experiment, and we highlight the new phenomenology introduced by the hydrodynamic model with respect to the Fourier-based description with the use of the analytical KCM solutions. Finally, the sixth section is devoted to concluding remarks.

\section{EFFECTIVE FOURIER FRAMEWORK}

To motivate the use of a generalized heat transport equation, it is important to first demonstrate the inability of an effective Fourier model to reproduce the detailed temporal information obtained with FDTR experiments $[13,14]$, in which a silicon substrate is heated with a sinusoidal laser pulse with frequency $f$; the thermal response is measured by a second laser and compared with the original heating wave. To achieve this, the substrate is covered by a transducer of gold (64 nm in height) that absorbs the laser power through electron excitation and releases it to the substrate. From the amplitude of the temperature oscillations $\Delta T$ in the transducer and the phase shift $\varphi$ between the temperature response and the heating function, information about the TBR between materials and the thermal transport in the substrate can be obtained.

The described experiment is very sensitive to the contact between the metal and the substrate. To reduce this effect, in [13], the authors include a thin layer of chromium between the gold and the silicon to reduce the TBR and hence make the experiment more sensitive to the substrate conduction. In the original work [13], a Kapitza interface boundary condition with conductance $210 \mathrm{MW} \mathrm{m}{ }^{-1} \mathrm{~K}^{-1}$ between an $\mathrm{Au} / \mathrm{Cr}$ transducer and the $\mathrm{Si}$ substrate is assumed. In this section, we use the same conductance as well as the other thermal properties for both domains reported in [13] at $311 \mathrm{~K}$.

In Fig. 1, we show the phase shift $\varphi$ and the temperature amplitude $\Delta T$ measurements compared with the analytical results obtained using Fourier's law in the substrate with different values of the thermal conductivity. The blue line represents the results obtained using the bulk value of the thermal conductivity for the substrate. It can be seen that for very small frequencies the predictions for both $\Delta T$ and $\varphi$ agree with the experimental data, but as the frequency increases, both curves deviate from the data. To fit to the data in the whole range of frequencies, it seems natural to assume a frequency-dependent thermal conductivity. To fit to the phase shift, the effective thermal conductivity must decrease with increasing frequency of the laser pulse beam, as shown in [13]. However, using this type of thermal conductivity leads to a poor fit to the 


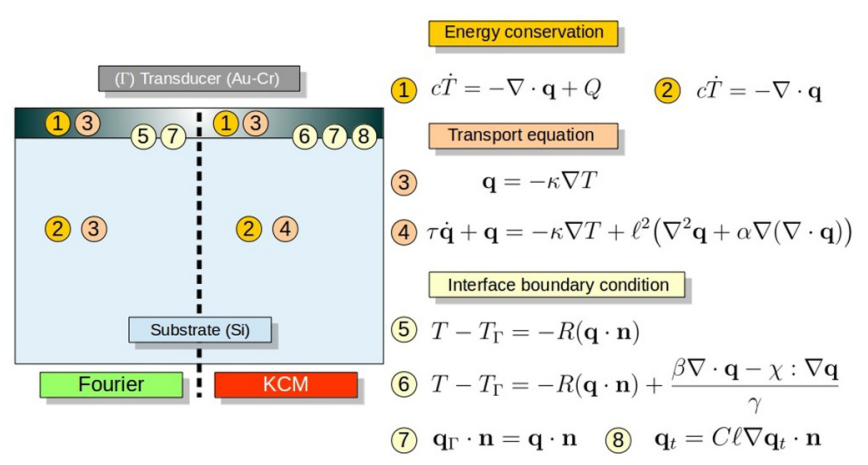

FIG. 2. Transport equations and interface boundary conditions used in Fourier and KCM for the heat flux $\mathbf{q}$ and the temperature $T$. The interface normal vector $\mathbf{n}$ points toward the semiconductor. The substrate heat flux tangential component is denoted by $\mathbf{q}_{t}$, and subindex $\Gamma$ refers to the transducer domain in the boundary conditions. A detailed explanation of KCM can be found in Appendix A, and the equation parameter values can be found in Appendix C.

temperature amplitude. To reproduce the reduction of the temperature amplitude $\Delta T$, the effective thermal conductivity for the substrate must increase with increasing frequency. In this case, the fit to the phase-shift curve becomes worse. The conclusion to this is that the use of a frequency-dependent effective thermal conductivity cannot simultaneously explain both experimental observables $\varphi, \Delta T$ at $311 \mathrm{~K}$.

\section{KINETIC COLLECTIVE MODEL}

For the aforementioned reasons, we now propose an alternative model to describe the experiment that makes use of the hydrodynamic heat transport equation for the Si substrate,

$$
\tau \dot{\mathbf{q}}+\mathbf{q}=-\kappa_{\mathrm{Si}} \nabla T+\ell^{2}\left[\nabla^{2} \mathbf{q}+\alpha \nabla(\nabla \cdot \mathbf{q})\right],
$$

where $\kappa_{\mathrm{Si}}$ is the bulk thermal conductivity, $\tau$ is the heat flux relaxation time, $\ell$ is the nonlocal length, and $\alpha$ is a dimensionless parameter. This generalized equation has been derived from the Boltzmann transport equation in the limit where normal collisions are dominant (collective regime) with $\alpha=2$ [26], and also when the resistive collisions are important by assuming an averaged phonon mode relaxation time with $\alpha=1 / 3$ [28]. Equation (1) captures the effect of momentum conservation due either to normal collisions, as in the collective regime, or to the relative absence of resistive collisions close to the boundaries at length scales smaller than the average phonon mean free path, even in the kinetic regime (resistive-dominant collisions). The KCM combines the influence of normal and resistive collisions through specific microscopic expressions for $\kappa, \ell$, and $\tau$ that interpolate from the kinetic and collective limits [21,29]. At the mesoscopic level of description, the nonlocal length $\ell$ and the relaxation time $\tau$ are intrinsic material properties that determine the length and time scales where the thermal perturbations are correlated, respectively.

The complete set of equations used in the KCM is described in Fig. 2 for the different domains and the interface. Comparison with the Fourier model has been included for illustration. A detailed explanation of the model and the equation parameters can be found in Appendix A.

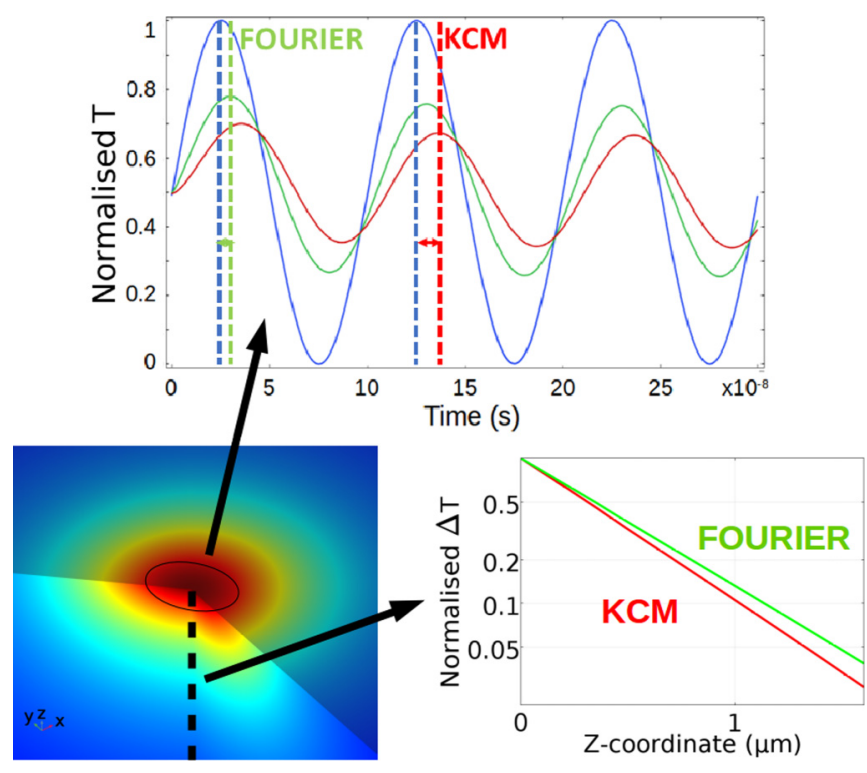

FIG. 3. Output obtained from the models using COMSOL MULTIPHYSICS for $f=100 \mathrm{MHz}$ and $T_{0}=311 \mathrm{~K}$. The top plot shows the heating energy density function (blue line) and the temperature evolution of the transducer surface according to bulk Fourier and KCM, respectively. The right-bottom plot shows the amplitude of the temperature oscillations along the cross-plane direction.

The hydrodynamic transport equation is only required to describe substrate conduction as heat is mainly carried by phonons with a large mean free path. For the transducer this change is not necessary as in metals heat is mainly carried by electrons, which have a significantly shorter mean free path.

For consistency with the substrate transport equation, nonlocal terms must be included in the interface boundary conditions. In Appendix B we provide a detailed derivation of the interface boundary condition by imposing energy conservation with the use of the corresponding substrate and transducer nonequilibrium distribution functions. The semiconductor phonon distribution function used is consistent with the transport equation (1) with $\alpha=1 / 3$ as shown in [28]. Therefore, we use this form of Eq. (1). For the rest of the parameters included in the equations, we use $a b$ initio calculated values independent of the geometry and the heating frequency. For the materials under study, the values are shown in Table I of Appendix C. The same temperature-dependent hydrodynamic parameters for silicon have been used in previous work [22] to predict the effective thermal conductivity of complex geometries in stationary situations.

Figure 3 displays the KCM and Fourier predictions for a given heating frequency using COMSOL MULTIPHYSICS. It shows that the nonlocal term included in the transport equation modifies the system thermal response with respect to Fourier. In the next section, we show that KCM captures most of the phenomena observed in the FDTR experiment.

\section{RESULTS}

The system of partial differential equations described in the previous section can be solved analytically using integral transforms (see Appendix D for details). Calculating 


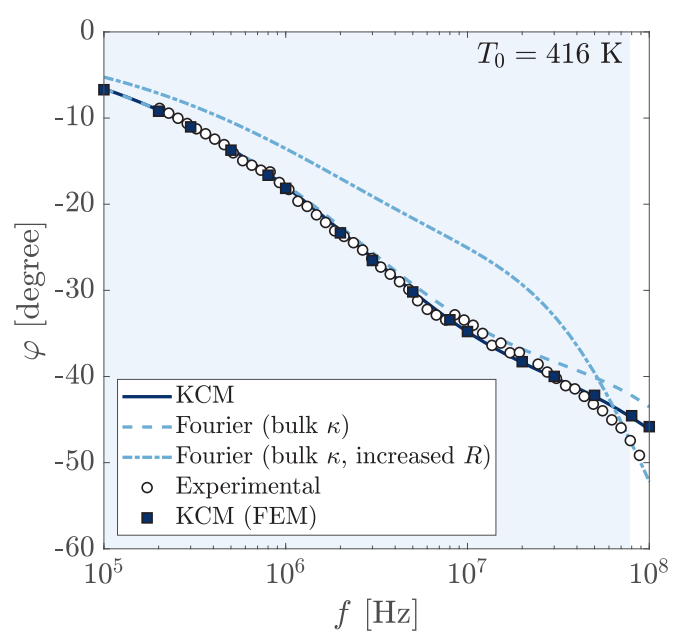

(a)

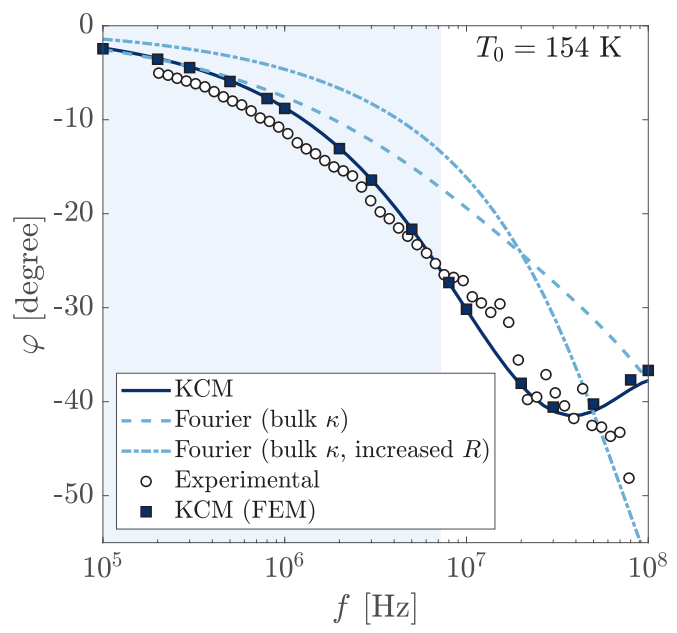

(c)

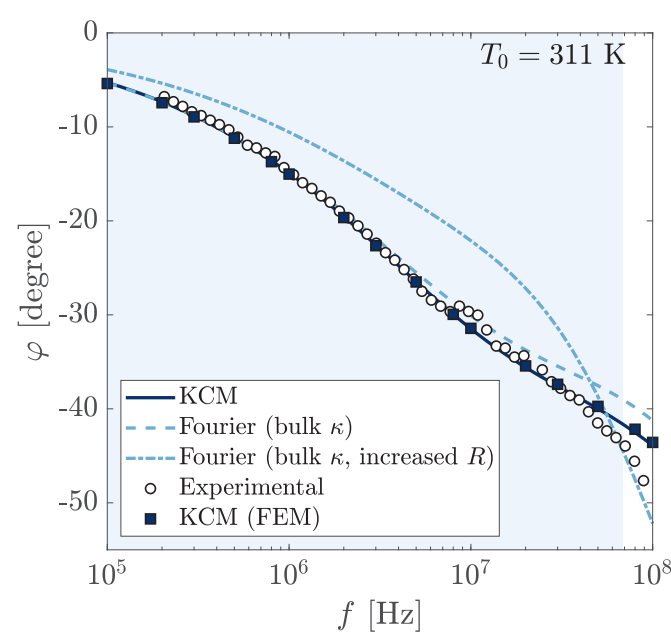

(b)

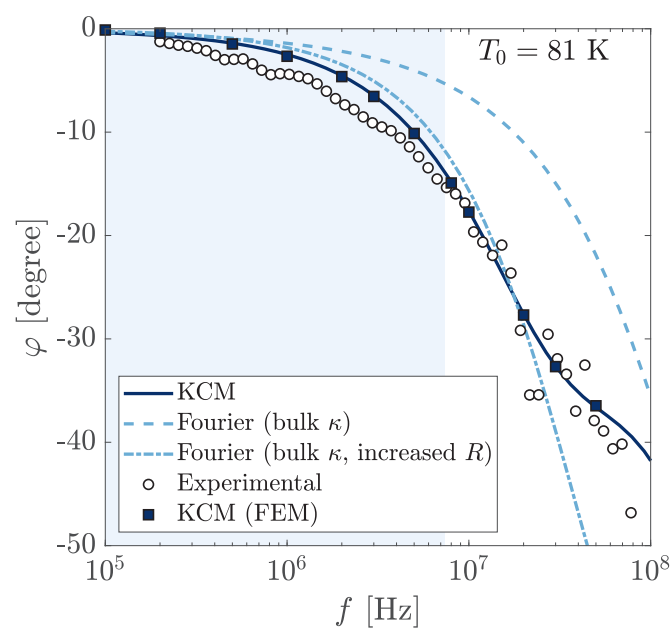

(d)

FIG. 4. Phase shift $\varphi$ as a function of frequency $f$ at (a) $T_{0}=416 \mathrm{~K}$, (b) $311 \mathrm{~K}$, (c) $154 \mathrm{~K}$, and (d) $81 \mathrm{~K}$. The KCM and Fourier (bulk $\kappa$ ) solutions use the thermal conductivity and the thermal boundary resistance $R$ reported in Table I (Appendix C). The Fourier (bulk $\kappa$, increased $R$ ) solution uses an enhanced thermal boundary resistance chosen to fit the high-frequency measurements, which leads to a poor fit at low frequencies. The agreement between the finite elements calculation and the KCM analytical solutions is excellent.

the change in surface temperature $\Delta T$ then amounts to performing numerical integration of the local temperature over a function with the form of the probe spot. The phase shift $\varphi$ can then be directly calculated from the argument of $\Delta T$. To support this analytical methodology, we also obtained numerical solutions via the FEM implementation presented in [22] using COMSOL MULTIPHYSICS.

In Fig. 4, we show the corresponding KCM results for the phase shift $\varphi$ at different temperatures 416, 311, 154, and $81 \mathrm{~K}$ compared with the experimental data [13] and the bulk Fourier prediction with the same thermal boundary resistance, $R$, as in KCM. For illustration, we also show that the bulk Fourier solutions with an increased TBR fitted to reproduce high-frequency measurements do not fit the low-frequency measurements. The normalized temperature oscillation amplitude $\Delta T$ is also compared in Fig. 5 at the experimentally available temperature: $311 \mathrm{~K}$. All quantities correspond to a weighted average across the surface of the transducer, computed using the Gaussian function of the probe beam as the weight according to the supplementary material of [14].
From Figs. 4 and 5 it can be observed that by using frequency-independent coefficients obtained from $a b$ initio calculations of natural bulk $\mathrm{Si}$, we obtain good agreement between experiments and the $\mathrm{KCM}$ for both $\varphi$ and $\Delta T$.

In previous work [22], it was shown that non-Fourier effects emerge in experiments involving small characteristic lengths comparable to the nonlocal length $\ell$. In the present case, the thermal penetration depth is the limiting length scale (see Sec. V for more details about the penetration depth). By increasing the heating frequency, the penetration depth is reduced and non-Fourier effects become significant. For illustration, we include in the figures a blue shading indicating the range of frequencies for which the classical thermal penetration depth $L_{\mathrm{Si}}=\sqrt{D_{\mathrm{Si}} /(\pi f)}$ is larger than three times the microscopic characteristic length $\sqrt{(1+\alpha)} \ell$ (where $D_{\mathrm{Si}}$ is the substrate thermal diffusivity and $\alpha=1 / 3)$.

Across the experimentally accessible range of frequencies, the memory term in the hydrodynamic equation (1) with coefficient $\tau$ and the hydrodynamic corrections in the interface condition (A6) only play important roles at temperatures 


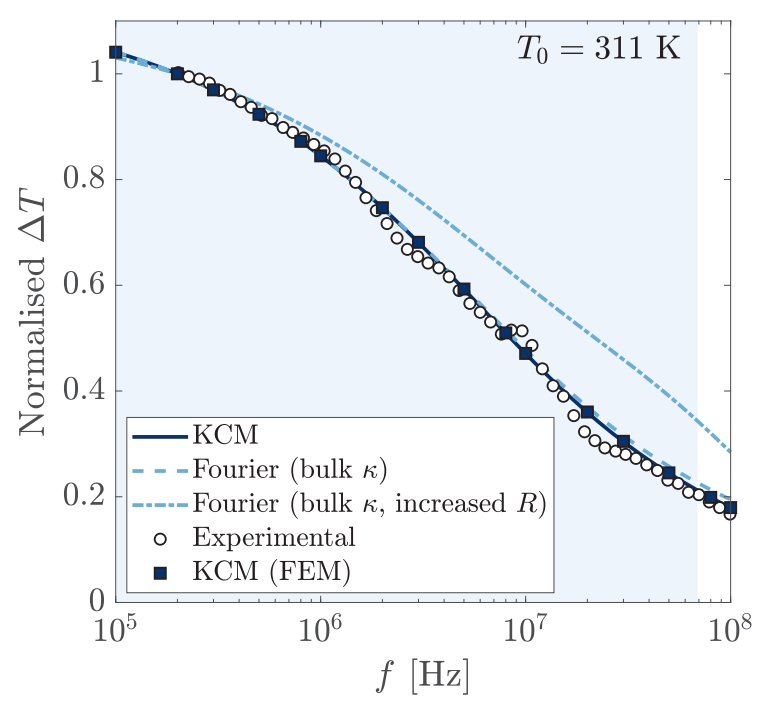

FIG. 5. Normalized temperature amplitude $\Delta T$ as a function of frequency $f$ at $T_{0}=311 \mathrm{~K}$.

below $200 \mathrm{~K}$ and provide small corrections. The inclusion of the viscous (nonlocal) term with coefficient $\ell^{2}$ in the heat transport equation is the main cause for the non-Fourier corrections.

Finally, a comment on the observed discrepancy at high temperatures and high frequencies $f>100 \mathrm{MHz}$ is in order. In the present work, the $\mathrm{Au} / \mathrm{Cr}$ transducer is modeled as a single domain with homogeneous properties. However, the presence of an interface between the 5-nm-thick chromium layer and the rest of the transducer influences the thermal response of the system at high frequencies, as suggested in [2]. In Appendix E we discuss the bulk Fourier model and the $\mathrm{KCM}$ considering detailed heat conduction in the transducer. Specifically, we show that by restricting the energy deposition to the chromium layer and including a $\mathrm{Cr}$-Au Kapitza interface thermal resistance, the discrepancy can be resolved at $T_{0}=311 \mathrm{~K}$.

\section{DISCUSSION}

The main effect of the nonlocal term of Eq. (1) is the modification of the thermal penetration depth. The analytical solution to the KCM (see Appendix D) sheds light on this previously unreported mechanism. Specifically, it reveals that there are two modes of thermal transport, which are analogous to pressure (P-mode) and shear ( $\mathrm{S}$-mode) waves in viscoelastic media. The P-mode captures longitudinal flux waves that are irrotational (as in the case of Fourier's law), whereas the S-mode captures transverse flux waves that are divergencefree (and thus temperature-conserving). The transverse nature of the S-modes means they can only be a feature of threedimensional heat conduction and would not be observed in one-dimensional models. Based on the analysis, we can define the penetration depths of the KCM P-modes and S-modes as $L_{\mathrm{P}}=1 / \operatorname{Re}\left\{\lambda_{\mathrm{p}}(0)\right\}$ and $L_{\mathrm{S}}=1 / \operatorname{Re}\left\{\lambda_{\mathrm{s}}(0)\right\}$, respectively, where $\lambda_{\mathrm{p}}$ and $\lambda_{\mathrm{s}}$ are defined by (D8) (see Fig. 6). The penetration depth $L_{\mathrm{S}}$ is proportional to the nonlocal length $\ell$. At low frequencies $\left(f \ll D_{\mathrm{Si}} / \ell^{2}\right)$, the penetration depth $L_{\mathrm{P}}$ is equivalent

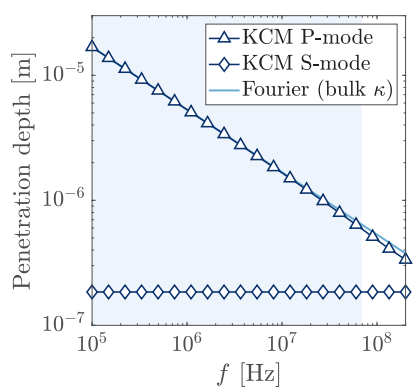

(a)

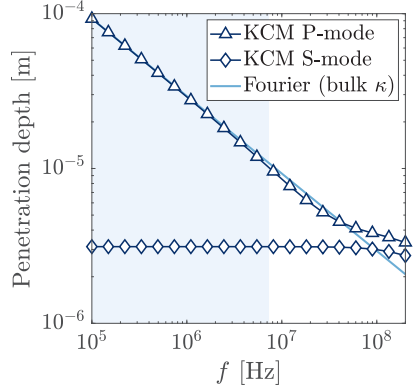

(b)
FIG. 6. The thermal penetration depths computed from Fourier and $\mathrm{KCM}$ at (a) $T_{0}=311 \mathrm{~K}$ and (b) $T_{0}=81 \mathrm{~K}$.

to $L_{\mathrm{Si}}$, the classical penetration depth for $\mathrm{Si}$ derived from Fourier's law. However, at high frequencies $\left(f \gg D_{\mathrm{Si}} / \ell^{2}\right)$, the penetration depth $L_{\mathrm{P}}$ becomes proportional to $\ell$ as well, with $L_{\mathrm{P}} / L_{\mathrm{S}} \rightarrow(\alpha+1)^{-1 / 2}$, indicating that the penetration depth of both modes can become comparable. The coupling of both modes at high frequencies causes deviations in the thermal response of the substrate with respect to the classical description based on Fourier's law. A detailed analysis of the onset of these hydrodynamic heat transport effects depending on the heating frequency can be found in Appendix F.

The non-Fourier behavior predicted by KCM provides a description of the experimental data with a fixed set of parameters at different temperatures. In contrast, the Fourier-based description requires an enhanced thermal boundary resistance $R$ in order to fit the high-frequency measurements of the phase shift, which leads to a poor fit at low frequencies, as can be seen in Fig. 4. In addition, the improved performance of the KCM allows for better modeling of the semiconductortransducer TBR. In previous works using Fourier's law, a TBR that is dependent on the size of the contact has been used to interpret similar experimental results [4]. However, the resistance $R$ is an intrinsic property determined only by the mismatch between the contact materials and the quality of the contact.

Assuming no defects in the contact area of the interface and diffusive phonon reflections, a lower bound for the thermal boundary resistance $R$ in the interface condition (A6) is $R_{\min }=\left(\gamma_{0}^{-1}+\gamma_{0_{\Gamma}}^{-1}\right) / 2$, with $\gamma_{0}$ and $\gamma_{0_{\Gamma}}$ depending only on the specific heat and group velocities of the phonon modes (a derivation of this lower bound using the hydrodynamic nonequilibrium distribution function can be seen in Appendix B). This thermal resistance arises from the phonon distribution function mismatch between chromium and silicon, and is in agreement with the diffuse mismatch model (DMM) [27]. Current fabrication processes do not allow perfect contact and hence the thermal resistance is larger than this lower bound. This is because the metal is sputtered on top of the substrate, which implies that the interface is not purely crystalline. We cannot quantitatively predict the value of $R$ because of the lack of knowledge of the interface defects. However, it is natural to expect that the correction due to interface imperfections is temperature-independent. Consequently, we fitted a temperature-independent scaling factor with respect to the lower bound $R / R_{\min }=2.71$ in order to reproduce 
the experimental measurements at low frequencies (which do not depend on hydrodynamic effects, i.e., the bulk Fourier and the KCM predictions coincide, as can be seen in Fig. 4). The rest of the terms in (A6) are higher-order corrections derived from the nonequilibrium phonon distribution function describing the substrate close to the interface, as shown in Appendix B. These terms cause only small corrections in the present results.

\section{CONCLUDING REMARKS}

We have detailed a multiscale hydrodynamic heat transport model that is able to predict FDTR experimental measurements using $a b$ initio calculated parameters. In contrast to previous interpretations based on an effective Fourier framework [13], by capturing the nonequilibrium effects induced by the interface and the nonhomogeneous heating, the model is able to reproduce both the amplitude of the temperature oscillations and the phase shift with the same set of geometryand frequency-independent coefficients. The analytical solutions to the KCM for the situation under study have been used to discuss the phenomenological interpretation of the hydrodynamic model by comparison with the Fourier bulk prediction. According to the KCM, the onset of non-Fouriertransport mechanisms that modify the penetration depth at high frequencies is the natural way to explain the experimental data, which cannot be fully described as an effective reduction of the thermal conductivity.

Special attention has been devoted to explaining the required boundary conditions. In particular, we have shown that the interface temperature-jump condition is obtained by imposing microscopic energy balance restrictions with use of the specific nonequilibrium distribution function accounting for nonlocal effects. The results show that by using values of the thermal boundary resistance proportional to the lower bound predicted by the DMM, the KCM is able to give remarkable predictions. In contrast, the Fourier-based models require large values of the thermal boundary resistance to fit the measurements at high frequencies, which do not fit the low-frequency range measurements [13].

The model was previously presented in [22] for stationary situations to calculate the effective thermal conductivity of silicon samples with an arbitrary geometry. Here we extended its use to transient situations including metal-semiconductor interfaces. Therefore, in this work we have shown how to integrate the hydrodynamic description of the substrate heat transport in the presence of contact metal domains. We have also found excellent agreement between the analytical KCM solutions and the numerical solutions obtained using the FEM implementation presented in [22].

\section{ACKNOWLEDGMENTS}

We acknowledge financial support by Spain's Ministerio de Ciencia, Innovación y Universidades under Grant No. RTI2018-097876-B-C22 (MCIU/AEI/FEDER, UE). M.G.H. received funding from the European Union's Horizon 2020 research and innovation programme under the Marie Skłodowska-Curie Grant Agreement No. 707658. T.G.M. ac- knowledges the support of Ministerio de Ciencia, Innovación y Universidades Grant No. MTM2017-82317-P.

\section{APPENDIX A: DESCRIPTION OF THE MODEL}

In this Appendix, we provide a full description of the $\mathrm{KCM}$ transport equations and boundary conditions required to predict the temperature $T$ and the heat flux $\mathbf{q}$ in the transducer (denoted by subindex $\Gamma$ ) and in the semiconductor.

\section{Transport equations}

Heat conduction in the transducer layer (mainly composed of gold with thickness $h=64 \mathrm{~nm}$ ) is described using conservation of energy along with Fourier's law,

$$
\begin{aligned}
c_{\Gamma} \dot{T}_{\Gamma}+\nabla \cdot \mathbf{q}_{\Gamma} & =Q, \\
\mathbf{q}_{\Gamma}+\kappa_{\Gamma} \nabla T_{\Gamma} & =0,
\end{aligned}
$$

where the dot denotes differentiation with respect to time, $c_{\Gamma}$ is the transducer specific heat, $\kappa_{\Gamma}$ is the transducer bulk thermal conductivity, and

$$
Q=Q_{0} \sin (2 \pi f t) \exp \left(\frac{-2 r^{2}}{r_{s}^{2}}\right)
$$

is the imposed heating energy density with $Q_{0}=1 \mathrm{~W} \mathrm{~m}^{-3}$, $r_{s}=3.2 \mu \mathrm{m}, r$ is the radial coordinate, and $f$ is the heating frequency of the laser pulse beam. Thermal transport in the substrate is modeled using conservation of energy and the hydrodynamic equation,

$$
\begin{aligned}
c_{\mathrm{Si}} \dot{T}+\nabla \cdot \mathbf{q} & =\mathbf{0}, \\
\tau \dot{\mathbf{q}}+\mathbf{q}+\kappa_{\mathrm{Si}} \nabla T & =\ell^{2}\left[\nabla^{2} \mathbf{q}+\alpha \nabla(\nabla \cdot \mathbf{q})\right],
\end{aligned}
$$

where $c_{\mathrm{Si}}$ is the substrate specific heat, $\kappa_{\mathrm{Si}}$ is the substrate bulk thermal conductivity, $\ell$ is the nonlocal length, $\tau$ is the heat flux relaxation time, and $\alpha$ is a dimensionless parameter. The microscopic expressions of these parameters for Si can be found elsewhere [21,29].

\section{Boundary conditions}

At the transducer free surface $(x=-h)$ insulation is imposed,

$$
\mathbf{q}_{\Gamma} \cdot \mathbf{n}=\mathbf{0},
$$

where $\mathbf{n}$ is the boundary normal vector. The temperature of the substrate base is fixed to the initial temperature $T_{0}$.

As indicated in [14], a $5 \mathrm{~nm}$ adhesion layer of chromium is introduced in the transducer base between the gold and the semiconductor to enhance the interface thermal conductance. We impose three conditions at the metal-silicon interface $(x=$ $0)$ : The first is continuity of the normal component of the heat flux:

$$
\mathbf{q}_{\Gamma} \cdot \mathbf{n}=\mathbf{q} \cdot \mathbf{n} .
$$

The second is a temperature-jump boundary condition that accounts for the phonon population mismatch between the metal and the semiconductor along with the nonequilibrium effects introduced by the interface,

$$
T-T_{\Gamma}=-R \mathbf{q} \cdot \mathbf{n}+\gamma^{-1}(\beta \boldsymbol{\nabla} \cdot \mathbf{q}-\chi: \nabla \mathbf{q}),
$$


where $\mathbf{n}$ points toward the semiconductor and $\gamma, \beta, \chi$ are $a b$ initio coefficients. The tensor $\chi$ is diagonal. The TBR value $R$ coincides with the DMM prediction with a temperatureindependent correction to account for the sample interface defects as mentioned in Sec. V. The derivation of this boundary condition with the use of the specific nonequilibrium distribution function consistent with (A3b) (assuming diffusive reflections at the interface) and the explicit microscopic expressions for these parameters can be found in Appendix B. The last two terms give only small corrections.

Finally, a slip boundary condition for the tangential components of the substrate heat flux $\mathbf{q}_{t}$ is required:

$$
\mathbf{q}_{t}=C \ell \nabla \mathbf{q}_{t} \cdot \mathbf{n} .
$$

The slip coefficient $C=\frac{1+p}{1-p}$ depends on the fraction of specularly reflected phonons $p$, which can be calculated using the wavelength of the phonon modes, the specific heats, and the roughness of the boundaries as discussed in [22]. In the present case, diffusive boundary reflections are expected, so that $p=0$ and $C=1$.

The values for all the parameters can be found in Table I of Appendix C.

\section{APPENDIX B: INTERFACE BOUNDARY CONDITIONS}

In [28], the derivation of the transport equation (A3b) for a semiconductor was carried out in the kinetic regime with an averaged phonon relaxation time using a perturbation expansion of the BTE around the nonequilibrium distribution obtained by the maximum entropy principle. The corresponding nonequilibrium distribution function $f$ reads

$$
\begin{aligned}
f= & f^{\mathrm{eq}}+\frac{3}{c v_{g}^{2}} \frac{\partial f^{\mathrm{eq}}}{\partial T} q_{i} v_{g i}+\frac{\tau_{R}}{c} \frac{\partial q_{i}}{\partial x_{i}} \frac{\partial f^{\mathrm{eq}}}{\partial T} \\
& -\frac{3 \tau_{R}}{c v_{g}^{2}} v_{g i} v_{g j} \frac{\partial q_{i}}{\partial x_{j}} \frac{\partial f^{\mathrm{eq}}}{\partial T}
\end{aligned}
$$

where $f^{\text {eq }}$ is the equilibrium distribution function, $v_{g}$ is the phonon group velocity, $c$ is the specific heat, and $\tau_{R}$ is an averaged relaxation time.

Here we assume that this nonequilibrium distribution function can be used to derive the appropriate boundary conditions at the interface using energy balance restrictions. Consider the interface normal vector $\mathbf{n}$ pointing toward the semiconductor. The balance equation for the energy flux at the interface implies that

$$
q_{x}=\int_{\Omega} f \hbar w v_{g x} d \mathbf{k}=\int_{\Omega^{-}} f^{-} \hbar w v_{g x} d \mathbf{k}+\int_{\Omega^{+}} f^{+} \hbar w v_{g x} d \mathbf{k},
$$

where $\mathbf{k}$ is the phonon wave vector, $\Omega$ denotes the whole wave-vector space, and $\Omega^{-}$and $\Omega^{+}$are the hemispherical wave-vector space satisfying $\mathbf{k} \cdot \mathbf{n}<\mathbf{0}$ and $\mathbf{k} \cdot \mathbf{n}>\mathbf{0}$, respectively. The normal direction is denoted by the index $x$.

The distribution function of the incident phonons toward the interface from the semiconductor $f^{-}$follows the nonequilibrium distribution function $f$. Moreover, the distribution function of reflected phonons $f^{+}$is composed by the specularly reflected ones following $f$ and the diffusively reflected phonons, which are in equilibrium at some contact temperature $T_{C}$. Accordingly,

$$
\begin{aligned}
f^{-} & =f, \\
f^{+}\left(v_{g x}\right) & =p f^{-}\left(-v_{g x}\right)+(1-p) f^{\mathrm{eq}}\left(T_{C}\right),
\end{aligned}
$$

where $p$ is the fraction of specularly reflected phonons (already introduced in [22]) and $T_{C}$ is an instrumental parameter to define the equilibrium situation of the diffusively reflected phonons in the interface going toward the semiconductor.

In the metal region, we assume Fourier heat transport. The corresponding nonequilibrium distribution function $f_{\Gamma}$ reads

$$
f_{\Gamma}=f^{\mathrm{eq}}+\frac{3}{c^{\Gamma}\left(v_{g}^{\Gamma}\right)^{2}} \frac{\partial f^{\mathrm{eq}}}{\partial T} q_{i} v_{g i}^{\Gamma} .
$$

Again, we impose an energy balance at the interface:

$$
q_{x}=\int_{\Omega} f_{\Gamma} \hbar w v_{g x} d \mathbf{k}=\int_{\Omega^{+}} f_{\Gamma}^{-} \hbar w v_{g x} d \mathbf{k}+\int_{\Omega^{-}} f_{\Gamma}^{+} \hbar w v_{g x} d \mathbf{k},
$$

where $f_{\Gamma}^{-}$and $f_{\Gamma}^{+}$are the incident and outgoing phonon distribution functions satisfying conditions (B3a) and (B3b), respectively. Notice that $T_{C}$ is also used to define the equilibrium distribution of the diffusively reflected phonons going toward the metal.

Assuming $p=0$ (diffusive reflections) and by using the nonequilibrium distribution functions (B1) and (B4) in the balance equations (B2) and (B5) with identifying $T_{C}$ we obtain the boundary condition,

$$
\begin{aligned}
T-T_{\Gamma}= & \left(\gamma_{0}^{-1}+\gamma_{0_{\Gamma}}^{-1}\right)(-\mathbf{q} \cdot \mathbf{n}+\boldsymbol{\epsilon} \cdot \mathbf{q}) \\
& +\gamma_{0}^{-1}(\beta \nabla \cdot \mathbf{q}-\nabla \mathbf{q}: \chi),
\end{aligned}
$$

where the $a b$ initio calculated coefficients read for $i=x, y, z$

$$
\begin{aligned}
\gamma_{0} & =\frac{v_{g}}{4} c, \\
\gamma_{0_{\Gamma}} & =\frac{v_{g}^{\Gamma}}{4} c^{\Gamma} \\
\epsilon_{i} & =\int_{\Omega^{-}} \hbar w v_{g x}^{k} \frac{3}{c^{k}\left(v_{g}^{k}\right)^{2}} \frac{\partial f^{\mathrm{eq}}}{\partial T} v_{g i}^{k} d \mathbf{k}=\frac{1}{2} \delta_{i x}, \\
\beta & =\int_{\Omega^{-}} \hbar w v_{g x}^{k} \frac{\tau_{R}^{k}}{c^{k}} \frac{\partial f^{\mathrm{eq}}}{\partial T} d \mathbf{k}, \\
\chi_{i j} & =\int_{\Omega^{-}} \hbar w v_{g x}^{k} \frac{3 \tau_{R}^{k}}{c^{k}\left(v_{g}^{k}\right)^{2}} \frac{\partial f^{\mathrm{eq}}}{\partial T} v_{g i}^{k} v_{g j}^{k} d \mathbf{k} .
\end{aligned}
$$

By introducing the coefficients in (B6), we obtain the boundary condition

$$
T-T_{\Gamma}=-\frac{1}{2}\left(\gamma_{0}^{-1}+\gamma_{0_{\Gamma}}^{-1}\right) \mathbf{q} \cdot \mathbf{n}+\gamma_{0}^{-1}(\beta \nabla \cdot \mathbf{q}-\nabla \mathbf{q}: \chi) .
$$

The coefficient $\left(\gamma_{0}^{-1}+\gamma_{0_{\Gamma}}^{-1}\right) / 2$ is the thermal boundary resistance for perfect contact (i.e., all of the top surface of the substrate is covered with chromium) assuming diffusive reflections. Therefore, it can be considered as a lower bound for the thermal resistance. To obtain an adequate boundary condition with a nonperfect contact area (A6), we adjust the 
TABLE I. Temperature-dependent parameter values.

\begin{tabular}{lcccc}
\hline \hline & $81 \mathrm{~K}$ & $154 \mathrm{~K}$ & $311 \mathrm{~K}$ & $416 \mathrm{~K}$ \\
\hline$c_{\Gamma}\left(\mathrm{KJ} \mathrm{m}^{-3} \mathrm{~K}^{-1}\right)$ & 2470 & 2470 & 2470 & 2470 \\
$\kappa_{\Gamma}\left(\mathrm{W} \mathrm{m}^{-1} \mathrm{~K}^{-1}\right)$ & 110 & 110 & 110 & 110 \\
$c_{\mathrm{Si}}\left(\mathrm{KJ} \mathrm{m}^{-3} \mathrm{~K}^{-1}\right)$ & 466 & 1061 & 1692 & 1840 \\
$\kappa_{\mathrm{Si}}\left(\mathrm{W} \mathrm{m}{ }^{1} \mathrm{~K}^{-1}\right)$ & 1260 & 315 & 150 & 109 \\
$\tau(\mathrm{ps})$ & 1002 & 148 & 42 & 27 \\
$\ell(\mathrm{nm})$ & 3127 & 1047 & 185 & 142 \\
$\left.\gamma_{0}(\mathrm{MW} \mathrm{m})^{-2} \mathrm{~K}^{-1}\right)$ & 359 & 732 & 1068 & 1145 \\
$\left.\gamma_{0_{\Gamma}}(\mathrm{MW} \mathrm{m})^{-2} \mathrm{~K}^{-1}\right)$ & 975 & 1801 & 2237 & 2313 \\
$\beta(\mathrm{nm})$ & -745 & -87 & -21 & -14 \\
$\chi_{x x}(\mathrm{~nm})$ & -1066 & -129 & -31 & -21 \\
$\chi_{z z}(\mathrm{~nm})$ & -586 & -66 & -16 & -11 \\
$\left.1 / R(\mathrm{MW} \mathrm{m})^{-2} \mathrm{~K}^{-1}\right)$ & 194 & 384 & 534 & 565 \\
\hline \hline
\end{tabular}

coefficient multiplying the normal heat flux, which we denote by $R$. The $R$ value (reported in Table I) is 2.71 times larger than the lower bound at each temperature. Consistently, the same correction is also applied to the resistance weighting the nonlocal term $\gamma^{-1}=2.71 \gamma_{0}^{-1}$. The actual contact area at the interface is unknown. Hence, we are not able to quantitatively validate this correction to account for a nonperfect contact area.

Finally, the slip boundary condition (A7) for the tangential heat flux at the interface can be derived in an analogous way (this is also shown in [28]).

\section{APPENDIX C: PARAMETER VALUES}

All the temperature-dependent parameters that appear in the transport equations and boundary conditions can be found in Table I. The tensor $\chi$ is diagonal with $\chi_{y y}=\chi_{z z}$, where $x$ is the interface normal direction pointing toward the substrate. To obtain the microscopic expression for $\gamma_{0}, \gamma_{0_{\Gamma}}, \beta, \chi$ we assumed the nonequilibrium distribution function (B1), which is consistent with the transport equation (A3b) with $\alpha=1 / 3$ as shown in [28]. For consistency, here we use this form of Eq. (A3b).

As reported in [14], the transducer has a thickness of $h=$ $64 \mathrm{~nm}$. The thickness of the Si substrate is $525 \mu \mathrm{m}$, which we take as infinite when deriving analytic solutions to the model.

\section{APPENDIX D: KCM ANALYTICAL SOLUTIONS}

\section{Solution to the full model}

The KCM presented in Sec. III and Appendix A can be solved in terms of Hankel transforms by first writing the heating energy density (A2) as $Q=Q_{0} I(r) e^{-i \omega t}$, where $I(r)=$ $\exp \left(-2 r^{2} / r_{s}^{2}\right)$ is the dimensionless profile of the Gaussian beam and $\omega=2 \pi f$ is the angular frequency of the laser. The temperature $T_{\Gamma}$ and normal component of the flux $q_{\Gamma, x} \equiv$ $\mathbf{q}_{\Gamma} \cdot \mathbf{n}$ in the transducer can be written as

$$
\begin{aligned}
T_{\Gamma} & =\int_{0}^{\infty} \tilde{T}_{\Gamma}(x ; k) \tilde{I}(k) e^{-i \omega t} J_{0}(k r) k d k, \\
q_{\Gamma, x} & =\int_{0}^{\infty} \tilde{q}_{\Gamma, x}(x ; k) \tilde{I}(k) e^{-i \omega t} J_{0}(k r) k d k,
\end{aligned}
$$

where $\tilde{I}$ is the Hankel transform of $I$,

$$
\begin{aligned}
\tilde{T}_{\Gamma} & =-\frac{B \cosh \left[\lambda_{\Gamma}(x+h)\right]}{\kappa_{\Gamma} \lambda_{\Gamma} \sinh \left(\lambda_{\Gamma} h\right)}+\frac{Q_{0}}{\kappa_{\Gamma} \lambda_{\Gamma}^{2}}, \\
\tilde{q}_{\Gamma, x} & =\frac{B \sinh \left[\lambda_{\Gamma}(x+h)\right]}{\sinh \left(\lambda_{\Gamma} h\right)},
\end{aligned}
$$

and $\lambda_{\Gamma}=\left(k^{2}-i \Lambda_{\Gamma}^{-2}\right)^{1 / 2}$, with $\Lambda_{\Gamma}=\left[\kappa_{\Gamma} /\left(c_{\Gamma} \omega\right)\right]^{1 / 2}$ representing a thermal penetration depth for the transducer (note that $L_{\Gamma}=2^{1 / 2} \Lambda_{\Gamma}$ is the classical penetration depth). The constant $B$ is determined through the jump condition for the temperature (A6), which requires the solution to the substrate problem.

The hydrodynamic model for heat conduction in the substrate is solved by eliminating the temperature from (A3b) to obtain

$$
\tau \ddot{\mathbf{q}}+\dot{\mathbf{q}}=D_{\mathrm{Si}} \nabla(\nabla \cdot \mathbf{q})+\ell^{2}\left[\nabla^{2} \dot{\mathbf{q}}+\alpha \nabla(\nabla \cdot \dot{\mathbf{q}})\right],
$$

where $D_{\mathrm{Si}}=\kappa_{\mathrm{Si}} / c_{\mathrm{Si}}$ is the bulk thermal diffusivity of the substrate. Using a Helmholtz decomposition of the flux, $\mathbf{q}=$ $\nabla \Phi+\nabla \times \mathbf{A}$, the hydrodynamic transport equation reduces to a system of equations for the scalar potential $\Phi$ and the vector potential A:

$$
\begin{aligned}
\tau \ddot{\Phi}+\dot{\Phi} & =D_{\mathrm{Si}} \nabla^{2} \Phi+\ell^{2}(\alpha+1) \nabla^{2} \dot{\Phi}, \\
\tau \dot{\mathbf{A}}+\mathbf{A} & =\ell^{2} \nabla^{2} \mathbf{A}, \\
\nabla \cdot \mathbf{A} & =\mathbf{0} .
\end{aligned}
$$

In the case of Fourier conduction, the scalar potential $\Phi$ is equivalent to the temperature $T$ and the two are related by $\Phi=-\kappa_{\mathrm{Si}} T$. However, in this context, thermal transport is analogous to viscoelastic wave propagation; note that (D3) is mathematically identical to the equations of motion for a Kelvin-Voigt viscoelastic material with linear damping. The scalar and vector potentials may therefore be interpreted as the amplitude of viscoelastic pressure and shear waves, respectively. The Helmholtz decomposition of the flux implies that the vector potential cannot affect the bulk temperature, hence the thermal shear waves are temperature-conserving. By introducing a streamfunction $\psi$, the problem for the vector potential can be simplified by writing $\mathbf{A}=\nabla \times(\psi \mathbf{n})$. The incompressibility condition (D4c) is automatically satisfied, and the problem for $\psi$ becomes

$$
\nabla^{2}\left(\tau \dot{\psi}+\psi-\ell^{2} \nabla^{2} \psi\right)=0
$$

The scalar potential and streamfunction are then given by

$$
\begin{aligned}
& \Phi=\int_{0}^{\infty} \tilde{\Phi}(k) \tilde{I}(k) e^{-\lambda_{\mathrm{p}}(k) x-i \omega t} J_{0}(k r) k d k, \\
& \psi=\int_{0}^{\infty} \tilde{\psi}(k) \tilde{I}(k) e^{-\lambda_{\mathrm{s}}(k) x-i \omega t} J_{0}(k r) k d k,
\end{aligned}
$$

where

$$
\begin{aligned}
& \tilde{\Phi}=\left[\frac{\lambda_{\mathrm{s}}\left(1+C \ell \lambda_{\mathrm{s}}\right)}{k^{2}\left(1+C \ell \lambda_{\mathrm{p}}\right)-\lambda_{\mathrm{s}} \lambda_{\mathrm{p}}\left(1+C \ell \lambda_{\mathrm{s}}\right)}\right] B, \\
& \tilde{\psi}=\left[\frac{1+C \ell \lambda_{\mathrm{p}}}{k^{2}\left(1+C \ell \lambda_{\mathrm{p}}\right)-\lambda_{\mathrm{s}} \lambda_{\mathrm{p}}\left(1+C \ell \lambda_{\mathrm{s}}\right)}\right] B,
\end{aligned}
$$


and the nonclassical inverse thermal penetration depths satisfy

$$
\begin{aligned}
& \lambda_{\mathrm{p}}^{2}=k^{2}-\frac{i(1-i \omega \tau)}{\Lambda_{\mathrm{Si}}^{2}\left[1-i(\alpha+1)\left(\ell / \Lambda_{\mathrm{Si}}\right)^{2}\right]}, \\
& \lambda_{\mathrm{s}}^{2}=k^{2}+\ell^{-2}(1-i \omega \tau),
\end{aligned}
$$

with $\Lambda_{\mathrm{Si}}=\left(D_{\mathrm{Si}} / \omega\right)^{1 / 2}$ and $\operatorname{Re}\left\{\lambda_{\mathrm{p}}\right\}>0$ and $\operatorname{Re}\left\{\lambda_{\mathrm{s}}\right\}>0$. From the conservation of energy (A3a), the substrate temperature is found to be

$$
T=\int_{0}^{\infty} \tilde{T}(k) \tilde{I}(k) e^{-\lambda_{\mathrm{p}}(k) x-i \omega t} J_{0}(k r) k d k,
$$

where $\tilde{T}$ is related to $\tilde{\Phi}$ through the expression

$$
\tilde{T}=\frac{i}{c_{\mathrm{Si}} \omega}\left(k^{2}-\lambda_{\mathrm{p}}^{2}\right) \tilde{\Phi} .
$$

By taking the Hankel transform of the jump condition (A6) and using the fact that $\chi$ is a diagonal tensor with $\chi_{y y}=\chi_{z z}$, we find that

$$
\begin{aligned}
\tilde{T}-\tilde{T}_{\Gamma}= & -R B+\gamma^{-1}\left(\chi_{x x}-\chi_{z z}\right) \lambda_{\mathrm{s}} k^{2} \tilde{\psi} \\
& +\gamma^{-1}\left[\left(\beta-\chi_{x x}\right) \lambda_{\mathrm{p}}^{2}+\left(\beta+\chi_{z z}\right) k^{2}\right] \tilde{\Phi},
\end{aligned}
$$

which holds at $x=0$. Substitution of (D2a), (D10), and (D7) into (D11) results in a linear equation for the constant $B$.

Having solved the governing equations, the change in (complex) surface temperature measured by a probe beam with the same radius as the pump beam can be calculated as [30]

$$
\Delta T=\frac{r_{s}^{2}}{4} \int_{0}^{\infty} \tilde{T}_{\Gamma}(-h ; k) e^{-r_{s}^{2} k^{2} / 4} k d k
$$

The phase shift $\varphi$ is given by $\varphi=-\arg \{\Delta T\}$. The analytical solutions for the KCM shown in Figs. 4-9 are obtained by performing numerical integration of (D12).

\section{Reduction to an effective Fourier model}

The solution in the case of three-dimensional bulk Fourier transport in both the transducer and substrate can be obtained in the limit $\omega \tau \ll 1$ and $\ell / \Lambda_{\mathrm{Si}} \ll 1$, in which case $\lambda_{\mathrm{p}} \simeq$ $\left(k^{2}-i \Lambda_{\mathrm{Si}}^{-2}\right)^{1 / 2}, \lambda_{\mathrm{s}} \simeq\left(k^{2}+\ell^{-2}\right)^{1 / 2}, \tilde{\Phi} \simeq-B / \lambda_{\mathrm{p}}, \tilde{\psi} \simeq 0$, and $\tilde{T} \simeq B /\left(\kappa_{\mathrm{Si}} \lambda_{\mathrm{p}}\right)$. The constant $B$ can then be determined from a simplified form of the condition (D11) given by $\tilde{T}-\tilde{T}_{\Gamma}=$ $-R B$ at $x=0$. This form is consistent with neglecting hydrodynamic contributions to the nonequilibrium distribution function (B1) used to derive (B8), i.e., taking $\tau_{R} \rightarrow 0$ then leads to $\beta \rightarrow 0$ and $\chi \rightarrow 0$.

The Fourier model that is obtained from this reduction is similar to the analytical Fourier solution derived by Cahill [30]. However, Cahill captures the laser heating through a boundary condition (identical to that in Appendix D4), whereas we capture the heating through a source term in the bulk equation (A1a) because the laser optical penetration length in the transducer is much larger than its height $h$. At small frequencies, the difference between surface and volumetric heating is indistinguishable because the generated heat is rapidly transported across the transducer, and the two solutions are in good agreement. Minor differences in the solutions appear at large frequencies. Consistently, if the transducer thickness $h$ is decreased, the temperature evolution in the transducer become faster and the two solutions converge across all frequencies.

\section{Reduction to a one-dimensional model}

Heat conduction becomes one-dimensional in the highfrequency limit given by $\omega \gg D_{\Gamma} / r_{s}^{2}$. However, it is insightful to examine the one-dimensional model outside of the high-frequency limit because closed-form expressions for the temperature amplitude and phase shift can be obtained. Upon invoking the assumption of one-dimensional heat flow, the surface temperature amplitude is given by

$$
\Delta T^{(1 \mathrm{D})}=\frac{i Q_{0}}{2 \omega c_{\Gamma}}\left(1-\zeta^{-1}\right),
$$

where

$$
\begin{aligned}
\zeta= & \left\{\frac{\left(c_{\Gamma} \kappa_{\Gamma} \omega\right)^{1 / 2}}{i^{1 / 2}}\left[R+\left(\frac{\beta-\chi_{x x}}{i^{1 / 2} \gamma \Lambda_{\mathrm{Si}}}\right) \mathcal{N}\right]+\left(\frac{c_{\Gamma}}{c_{\mathrm{Si}}} \frac{\Lambda_{\Gamma}}{\Lambda_{\mathrm{Si}}}\right) \mathcal{N}\right\} \\
& \times \sinh \left(\frac{h}{i^{1 / 2} \Lambda_{\Gamma}}\right)+\cosh \left(\frac{h}{i^{1 / 2} \Lambda_{\Gamma}}\right) .
\end{aligned}
$$

The frequency-dependent function $\mathcal{N}$ captures non-Fourier effects due to bulk hydrodynamic thermal transport and is defined by

$$
\mathcal{N}=\left[\frac{1-i \omega \tau}{1-i(\alpha+1)\left(\ell / \Lambda_{\mathrm{Si}}\right)^{2}}\right]^{1 / 2} .
$$

The one-dimensional effective Fourier model can therefore be obtained from (D14) by setting $\mathcal{N}=1$ and $\beta=\chi_{x x}=0$ (see Appendix D 2). In the thin-film limit $h / \Lambda_{\Gamma} \ll 1$, Eq. (D14) simplifies to

$$
\begin{aligned}
\zeta= & 1+i^{-1 / 2}\left(\frac{c_{\Gamma}}{c_{\mathrm{Si}}} \frac{h}{\Lambda_{\mathrm{Si}}}\right) \mathcal{N} \\
& -c_{\Gamma} \omega h\left[i R+i^{1 / 2}\left(\frac{\beta-\chi_{x x}}{\gamma \Lambda_{\mathrm{Si}}}\right) \mathcal{N}\right] .
\end{aligned}
$$

To interpret the one-dimensional solutions for the phase shift shown in Fig. 8, it is useful to consider a further limit whereby $h / \Lambda_{\mathrm{Si}} \ll 1, c_{\Gamma} \omega h / \gamma \ll 1$, and $\left(\beta-\chi_{x x}\right) / \Lambda_{\mathrm{Si}} \ll 1$. These inequalities are true for frequencies on the order of $10 \mathrm{MHz}$ at both 81 and $311 \mathrm{~K}$. Since $\zeta$ is close to unity in this limit, the expression for the surface temperature amplitude reduces to

$$
\Delta T^{(1 \mathrm{D})}=\left(\frac{i}{\kappa_{\mathrm{Si}} c_{\mathrm{Si}} \omega}\right)^{1 / 2} \frac{Q_{0} h \mathcal{N}}{2}+\frac{Q_{0} h R}{2} .
$$

Equation (D17) reveals that for Fourier conduction $(\mathcal{N}=1)$ and small thermal boundary resistance $(R \rightarrow 0)$, the phase shift is a constant across all frequencies, $\varphi=-\pi / 4$. For finite values of $R$, the role of thermal boundary resistance is to increase the phase shift and bring it closer to zero. The good agreement between the one-dimensional Fourier and hydrodynamic models shown in Fig. 8 for frequencies less than $1 \mathrm{MHz}$ means that hydrodynamic transport is not relevant here. Thus, the increase in phase shift above $-45^{\circ}$ must be due to thermal boundary resistance. 


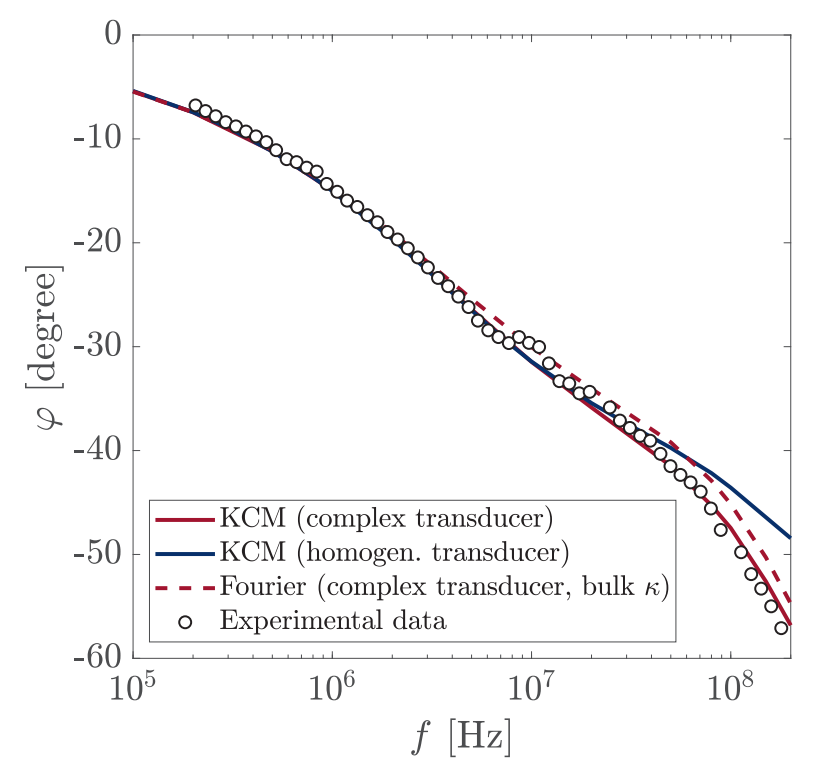

FIG. 7. Phase shift $\varphi$ at $T_{0}=311 \mathrm{~K}$ as predicted from hydrodynamic and Fourier models that consider the complex heat conduction in the $\mathrm{Au} / \mathrm{Cr}$ transducer (purple line). The blue line corresponds to a numerical solution of the hydrodynamic model in which the $\mathrm{Au} / \mathrm{Cr}$ transducer is modeled as a single homogeneous layer as in Fig. 4(b).

\section{A single-layer model for substrate conduction}

To isolate the role of hydrodynamic conduction in the experiments, we consider a simplified model consisting of a single layer of silicon, thereby removing the transducer and neglecting any effects due to thermal boundary resistance. The pump laser is assumed to impart a thermal flux of the form $\mathbf{q} \cdot \mathbf{n}=q_{0} I(r) e^{-i \omega t}$ at the surface of the Si layer, which is located at $x=0$. The governing equations consist of (A3)-(A7); however, the temperature jump condition (A6) is replaced with the surface-flux condition. The model can be analyzed in the same manner as before. The solutions for the substrate temperature and flux, in terms of the scalar potential and streamfunction, are the same and given by (D7)-(D10). However, the constant $B$ that appears in these solutions is now given by $B=q_{0}$. As a result, the expression for the measured

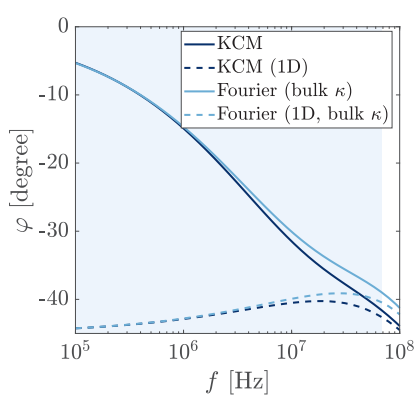

(a)

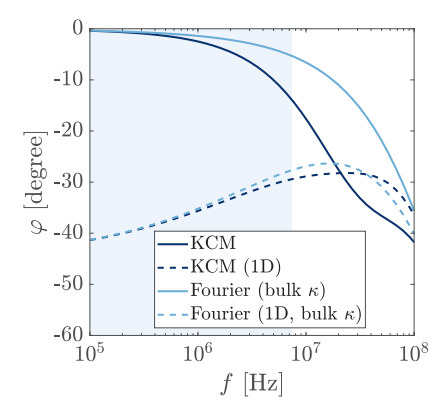

(b)
FIG. 8. The phase shift $\varphi$ computed from one- and threedimensional Fourier-based and KCM models at (a) $T_{0}=311 \mathrm{~K}$ and (b) $T_{0}=81 \mathrm{~K}$. surface temperature simplifies to

$$
\Delta T=-\left(\frac{r_{s}^{2}}{4 \kappa_{\mathrm{Si}}}\right) \mathcal{N}^{2} \int_{0}^{\infty} \tilde{\Phi}(k) e^{-r_{s}^{2} k^{2} / 4} k d k
$$

The high-frequency, one-dimensional limit of (D18) is given by

$$
\Delta T^{(1 \mathrm{D})}=\left(\frac{i}{\kappa_{\mathrm{Si}} c_{\mathrm{Si}} \omega}\right)^{1 / 2} \frac{q_{0} \mathcal{N}}{2},
$$

which coincides with the first term of the thin-film limit of the two-layer model (D17) once $q_{0}$ is identified with $Q_{0} h$. The corresponding phase shift is given by

$$
\varphi^{(1 \mathrm{D})}=-\frac{\pi}{4}-\frac{1}{2} \arctan \left(\frac{(\alpha+1) \ell^{2}}{\Lambda_{\mathrm{Si}}^{2}}\right)+\frac{1}{2} \arctan (\omega \tau) .
$$

Thus, nonlocal effects will decrease the phase shift below the Fourier prediction of $-\pi / 4$, whereas resistive effects increase the phase shift toward zero. Interestingly, the nonclassical contributions cancel out at high frequencies, recovering the Fourier result.

\section{APPENDIX E: INFLUENCE OF COMPLEX HEAT TRANSPORT IN THE METAL TRANSDUCER}

As shown in Sec. IV, most of the FDTR measurements from [14] can be explained assuming an homogeneous transducer and refined transport equations for the substrate heat conduction. However, some discrepancy is observed at very high frequencies, $f>100 \mathrm{MHz}$, for $T_{0}=311$ and $416 \mathrm{~K}$ This can be explained by assuming that the energy deposition by the laser is restricted to the 5-nm-thick chromium layer due to the very weak electron-phonon coupling in $\mathrm{Au}$ as suggested in [2]. In addition, an extra Kapitza interface thermal resistance between the chromium layer and the rest of the transducer is expected. In Fig. 7 we show the results using the corresponding COMSOL simulation assuming Fourier and hydrodynamic heat transport in the substrate, respectively. The thermal conductance value used for the $\mathrm{Au}-\mathrm{Cr}$ interface is $2 \mathrm{GW} \mathrm{m} \mathrm{m}^{-1} \mathrm{~K}^{-1}$, which is in reasonable agreement with previous work [2]. Therefore, complex heat transport in the transducer is a possible explanation for the extremely large phase shift measured at $f>100 \mathrm{MHz}$.

This refinement is only relevant at very high frequencies and hence does not significantly modify the phase-shift predictions at the experimentally accessible range of frequencies at low temperatures. Furthermore, it only causes minor corrections to the obtained amplitude of the temperature oscillations. In particular, the result presented in Sec. II is qualitatively the same.

\section{APPENDIX F: ANALYSIS OF THE ONSET OF HYDRODYNAMIC HEAT TRANSPORT MECHANISMS}

In this Appendix, we compare the phase shift obtained from one- and three-dimensional models based on Fourier's law and the hydrodynamic transport equation. In the Fourier models (also included in Figs. 4 and 5), the interface condition (A6) is replaced by the standard Kapitza boundary condition 


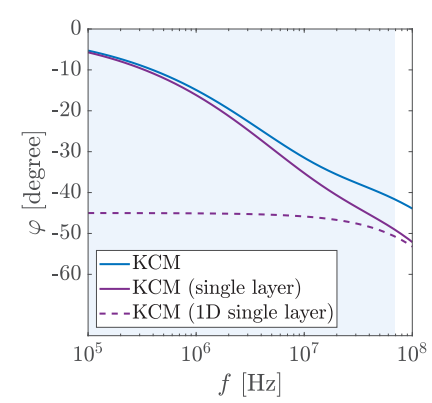

(a)

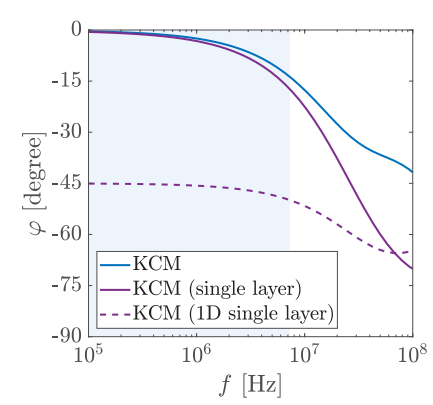

(b)
FIG. 9. Comparison of single-layer (substrate only) models with the full $\mathrm{KCM}$ model (substrate and transducer) for (a) $T_{0}=311 \mathrm{~K}$ and (b) $T_{0}=81 \mathrm{~K}$.

$T-T_{\Gamma}=-R \mathbf{q} \cdot \mathbf{n}$. The simplified form of the temperaturejump condition ensures that nonequilibrium hydrodynamic effects are consistently neglected in the Fourier model (see Appendix D 2).

The results of the comparisons when $T_{0}=311 \mathrm{~K}$ are shown in Fig. 8(a). Heat conduction is strongly threedimensional until frequencies on the order of $100 \mathrm{MHz}$ are reached. In principle, both modes of thermal transport can be active when heat conduction is three-dimensional; however, the good agreement between the KCM and Fourier models for frequencies up to $5 \mathrm{MHz}$ indicates that nonclassical transport mechanisms in the bulk do not play an important role in this regime. At $5 \mathrm{MHz}$, the $\mathrm{KCM}$ and Fourier models begin to diverge, highlighting the onset of hydrodynamic conduction mechanisms, which in this case are dominated by nonlocal effects. This is deduced from the relatively constant value of the penetration depth of the KCM S-modes (see Fig. 6), which implies that $\omega \tau \ll 1$ and thus hydrodynamic resistive effects are negligible across all frequencies, and the divergence of the P-mode penetration depth from the Fourier estimate. Due to the small penetration depth of the S-modes, these modes are highly localized and do not propagate into the bulk. Therefore, the three-dimensional conduction that occurs within the bulk in the frequency band 5-100 MHz is dominated by KCM P-modes. From $100 \mathrm{MHz}$ onward, heat conduction becomes one-dimensional. To summarize, there are three main conduction regimes when $T_{0}=311 \mathrm{~K}$ : (i) three-dimensional Fourier transport, (ii) three-dimensional hydrodynamic transport (dominated by nonlocal effects and interface resistance), and (iii) one-dimensional hydrodynamic transport (also dominated by nonlocal effects and interface resistance).

At a lower temperature of $T_{0}=81 \mathrm{~K}$, additional conduction mechanisms become relevant, as shown in Fig. 8(b). Nonlocal effects become particularly strong as the frequency is increased beyond $10 \mathrm{MHz}$, as can be seen from the marked departure of the P-mode penetration depth $L_{\mathrm{P}}$ from the classical penetration depth $\Lambda_{\mathrm{Si}}$. As the frequency increases beyond $50 \mathrm{MHz}$, the penetration depths of the P-and S-modes become comparable, and resistive hydrodynamic effects are activated (as seen from the variation in $L_{\mathrm{S}}$ with frequency).

To further investigate the role of thermal boundary resistance, in Fig. 9 we compare solutions to the single-layer model considering both hydrodynamic and Fourier conduction. At $311 \mathrm{~K}$, the monotonic decrease of the phase shift computed from the one-dimensional model implies that resistive effects are negligible. The good agreement between the single-layer and full KCM solutions at small frequencies reflects the relatively small influence of thermal boundary resistance in this regime. For a lower temperature of $81 \mathrm{~K}$, the large disparity between the single-layer and full KCM solution suggests that interface resistance plays an especially strong role at this temperature. The one-dimensional solution for the phase shift indicates that hydrodynamic conduction is first dominated by nonlocal effects, and then resistive effects become equally important.
[1] M. E. Siemens, Q. Li, R. Yang, K. A. Nelson, E. H. Anderson, M. M. Murnane, and H. C. Kapteyn, Nat. Mater. 9, 26 (2010).

[2] R. B. Wilson and D. G. Cahill, Nat. Commun. 5, 5075 (2014).

[3] Y. Hu, L. Zeng, A. J. Minnich, M. S. Dresselhaus, and G. Chen, Nat. Nanotechnol. 10, 701 (2015).

[4] K. M. Hoogeboom-Pot, J. N. Hernandez-Charpak, X. Gu, T. D. Frazer, E. H. Anderson, W. Chao, R. W. Falcone, R. Yang, M. M. Murnane, H. C. Kapteyn, and D. Nardi, Proc. Natl. Acad. Sci. (USA) 112, 4846 (2015).

[5] A. Ziabari, P. Torres, B. Vermeersch, Y. Xuan, X. Cartoixà, A. Torelló, J.-H. Bahk, Y. R. Koh, M. Parsa, P. D. Ye, F. X. Alvarez, and A. Shakouri, Nat. Commun. 9, 255 (2018).

[6] C. Hua, L. Lindsay, X. Chen, and A. J. Minnich, Phys. Rev. B 100, 085203 (2019).

[7] G. Romano, A. M. Kolpak, J. Carrete, and D. Broido, Phys. Rev. B 100, 045310 (2019).

[8] G. Romano, K. Esfarjani, D. A. Strubbe, D. A. Broido, and A. M. Kolpak, Phys. Rev. B 93, 035408 (2016).
[9] J. A. Johnson, A. A. Maznev, J. Cuffe, J. K. Eliason, A. J. Minnich, T. Kehoe, C. M. S. Torres, G. Chen, and K. A. Nelson, Phys. Rev. Lett. 110, 025901 (2013).

[10] K. T. Regner, J. P. Freedman, and J. A. Malen, Nanoscale Microscale Thermophys. Eng. 19, 183 (2015).

[11] T. D. Frazer, J. L. Knobloch, K. M. Hoogeboom-Pot, D. Nardi, W. Chao, R. W. Falcone, M. M. Murnane, H. C. Kapteyn, and J. N. Hernandez-Charpak, Phys. Rev. Appl. 11, 24042 (2019).

[12] A. Giri and P. E. Hopkins, Adv. Funct. Mater. 1903857 (2019).

[13] K. T. Regner, D. P. Sellan, Z. Su, C. H. Amon, A. J. H. McGaughey, and J. A. Malen, Nat. Commun. 4, 1640 (2013).

[14] K. T. Regner, S. Majumdar, and J. A. Malen, Rev. Sci. Instrum. 84, 064901 (2013).

[15] Y. K. Koh and D. G. Cahill, Phys. Rev. B 76, 075207 (2007).

[16] J. L. Braun, D. H. Olson, J. T. Gaskins, and P. E. Hopkins, Rev. Sci. Instrum. 90, 024905 (2019).

[17] D. Rodin and S. K. Yee, Rev. Sci. Instrum. 88, 014902 (2017).

[18] J. Liu, J. Zhu, M. Tian, X. Gu, A. Schmidt, and R. Yang, Rev. Sci. Instrum. 84, 034902 (2013). 
[19] B. Vermeersch, A. M. S. Mohammed, G. Pernot, Y. R. Koh, and A. Shakouri, Phys. Rev. B 91, 085203 (2015).

[20] B. Vermeersch, J. Carrete, N. Mingo, and A. Shakouri, Phys. Rev. B 91, 085202 (2015).

[21] P. Torres, A. Ziabari, A. Torelló, J. Bafaluy, J. Camacho, X. Cartoixà, A. Shakouri, and F. X. Alvarez, Phys. Rev. Mater. 2, 076001 (2018).

[22] A. Beardo, M. Calvo-Schwarzwälder, J. Camacho, T. G. Myers, P. Torres, L. Sendra, F. X. Alvarez, and J. Bafaluy, Phys. Rev. Appl. 11, 034003 (2019).
[23] X. Li and S. Lee, Phys. Rev. B 97, 094309 (2018).

[24] S. Lee, D. A. Broido, K. Esfarjani, and G. Chen, Nat. Commun. 6, 6290 (2015).

[25] A. Cepellotti and N. Marzari, Nano Lett. 17, 4675 (2017).

[26] R. A. Guyer and J. A. Krumhansl, Phys. Rev. 148, 766 (1966).

[27] E. T. Swartz and R. O. Pohl, Appl. Phys. Lett. 51, 2200 (1987)

[28] Y. Guo and M. Wang, Phys. Rev. B 97, 035421 (2018).

[29] P. Torres, A. Torelló, J. Bafaluy, J. Camacho, X. Cartoixà, and F. X. Alvarez, Phys. Rev. B 95, 165407 (2017).

[30] D. G. Cahill, Rev. Sci. Instrum. 75, 5119 (2004). 